\title{
POPULISMO, NAZIONALISMO E RELIGIONE TRA I POLITICI CRISTIANI LIBANESI DAL 2005 AL 2019
}

di Francesco Mazzucotelli

\section{Introduzione}

In questo articolo intendo evidenziare alcuni fenomeni che attingono ai registri del populismo e del nazionalismo nel campo politico cristiano libanese. L'arco temporale che prendo in considerazione va dalla primavera del 2005, quando il rientro nell'arena politica di Michel Aoun e Samir Ja'ja' segna un superamento della fase di frustrazione e disincanto (ihba a t) che aveva fatto seguito all'accordo di al-Ta'if e all'imposizione della pax syriana ${ }^{1}$, fino ai postumi delle elezioni parlamentari del 2018, soffermandomi in particolare sulla composizione delle liste elettorali e sui risultati nei collegi Monte Libano 1, Beirut 1, Nord Libano 3, Monte Libano 2 e Beqaa 1, ossia nei collegi con una maggioranza demografica cristiana ${ }^{2}$.

\section{Università di Pavia.}

1 S. HaugbøLle, The Secular Saint. Iconography and Ideology in the Cult of Bashir Jumayil, in A. BANDAK, M. BILlE (eds.), "Politics of Worship in the Contemporary Middle East: Sainthood in Fragile States", Leiden, Brill, 2013, pp. 191-212

2 Rimando all'Appendice per la composizione delle liste ammesse alla ripartizione dei seggi su base proporzionale in ogni collegio. Il sistema elettorale utilizzato nel 2018 ha conservato la ripartizione dei seggi su base contemporaneamente confessionale e territoriale, introducendo però un meccanismo proporzionale all'interno di ciascun collegio. Oltre a una presenza quasi continuativa tra il 2008 e il 2010, in occasione del dottorato di ricerca, per questo articolo mi baso su interviste, conversazioni informali, osservazioni partecipate e visite realizzate in Libano nel settembre 2012, settembre 2014, giugno 2015, gennaio 2017. Mi baso inoltre su contenuti apparsi su piattaforme di comunicazione e canali televisivi libanesi.

In questo articolo viene usata la traslitterazione scientifica secondo il sistema DIN 31635 per i termini del lessico politico, e una traslitterazione semplificata senza segni 
Dal punto di vista concettuale, mi rifaccio alla nozione di "nazionalismo cristiano" proposta da Felsch ${ }^{3}$, definibile come un sostrato ideologico più o meno manifestamente espresso che riprende le istanze di eccezionalismo e particolarismo che hanno attraversato la sfera pubblica cristiana libanese nel corso del ventesimo secolo. Per quanto declinato in maniera variegata e non sempre coerente, questo sostrato si basa sul presupposto che l'identità del Libano risieda essenzialmente nella sua storia di nazione cristiana, e che i cristiani abbiano di conseguenza un diritto storico di preminenza politica nella determinazione delle scelte strategiche di governo. Il nazionalismo cristiano libanese, come cerco di evidenziare in questo articolo, punta pertanto al mantenimento di uno stato a trazione cristiana, con una forte identità religiosa (cristiana), forti autonomie per le istituzioni confessionali, e anche con un tentativo di demarcazione territoriale delle aree a predominanza cristiana.

Non è mia intenzione riprendere qui la storia della genesi del nazionalismo libanese, e in particolare del "fenicismo" come mitologia delle origini utilizzata per legittimare un progetto di edificazione di identità nazionale alternativo a quelli del panarabismo e del nazionalismo pansiriano ${ }^{4}$. Mi preme tuttavia sottolineare come l'incubazione di quel discours libaniste studiato da Sassine ${ }^{5}$, ossia la genesi dell'idea nazionale libanese, abbia avuto luogo prevalentemente in ambiti cristiani, e più specificamente maroniti, per la protezione degli interessi politici ed economici della componente maronita, e attingendo a un immaginario e a un vocabolario in larga parte basati sulla tradizione ma-

diacritici per i nomi di luoghi e di persone. Questa versione semplificata in alcuni differisce dalle trascrizioni fonetiche francesizzanti (al-Jumayyil/Gémayel, Ja'ja'/Geagea, Junbulat/Jumblatt).

3 M. Felsch, The Rise of Christian Nationalism in Lebanon, in M. FelsCh, M. WäHLISCH (eds.), "Lebanon and the Arab Uprisings: In the Eye of the Hurricane", New York, Routledge, 2016, pp. 70-86.

4 A. Kaufman, Phoenicianism: The Formation of an Identity in Lebanon in 1920, in "Middle Eastern Studies", n. 1, 2001, pp. 173-194.

K. FIRro, Lebanese Nationalism versus Arabism: From Bulus Nujaym to Michel Chiha, in "Middle Eastern Studies", n. 5, 2004, pp. 1-27.

N. Dot-PoullLard, Sur les frontières: le Parti syrien national social entre idéologie unitaire et États-nation, in A. Bozzo, P.-J. LuIZARD (dir.), "Vers un nouveau Moyen-Orient? États arabes en crise entre logiques de division et sociétés civile", Roma: Roma TrE-Press, 2016, pp. 209-225.

5 F. SAssine, Le libanisme maronite. Contributions à l'étude d'un discours politique, Paris, Université de Paris Sorbonne, 1979, tesi di dottorato. 
ronita, a partire dai riferimenti biblici ${ }^{6}$. In questo discorso nazionalista prevale un forte determinismo geografico, che sembra individuare nel paesaggio un vero e proprio spirito della nazione ${ }^{7}$, e un'idea organicistica del corpo politico, che può essere rinnovato attraverso una guida carismatica e un costante ancoraggio alla propria memoria e al proprio suolo. Questa visione si basa nettamente sul pensiero di Maurice Barrès e sul concetto di nationalisme intégral, teorizzato da Charles Maurras e dai circoli dell'Action Françaises, e recepito in Libano per il tramite degli istituti formativi gesuiti 9 . Come ha dimostrato Bawardi10, il discorso nazionalista "libanista" si è sviluppato negli anni del Mandato francese e dopo l'indipendenza come un progetto culturale che ha usato molteplici strumenti, tra cui la fruizione di musei e siti archeologici, la letteratura in prosa e in poesia, i festival artistici, i racconti per l'infanzia e la musica per un obiettivo precipuamente politico, ossia la mobilitazione politica in sostegno di un'idea di nazione libanese fondata sulle sue radici immaginate fenicie e cristiane.

Non è neppure mia intenzione riprendere qui la cronologia politica libanese dal "Patto nazionale" del 1943 fino alla guerra civile. Mi sembra tuttavia fondamentale ricordare come l'ascesa della sinistra radicale, caratterizzata dall'intersezione tra marxismo, maoismo, terzomondismo e difesa della causa palestinese, ben riassunta da Maasri nel suo recente libro"11, abbia per converso stimolato non solo una radicalizzazione del campo politico cristiano, a cominciare dalla triplice

6 C.Hakim, The Origins of the Lebanese National Idea: 1840-1920, Berkeley, University of California Press, 2013.

7 R. Choueiri, Les sites paysagers de la mémoire du Liban, Baabda: Felix Béryte, 2012.

8 A. Kaufman, From "La Colline inspirée" to "La Montagne inspirée": Maurice Barrès and Lebanese Nationalism, in M. AвітвоL (ed.), "France and the Middle East: Past, Present, and Future", Jerusalem, Hebrew University Magnes Press, 2004, pp. 225-246. B. HeYBerger, Saint Charbel Makhlouf, ou la consécration de l'identité maronite, in C. MAYeur-Jouen (ed.), Saints et héros du Moyen-Orient contemporain, Paris, Maisonneuve et Larose, 2003, pp. 139-159.

9 R. HerzsteIn, Les pères jésuites et les Maronites du Mont-Liban: l'Université SaintJoseph de Beyrouth, in "Histoire et missions chrétiennes", n. 1, 2009, pp. 149-175.

${ }^{10}$ B. BAWARDI, The Lebanese-Phoenician Nationalist Movement: Literature, Language and Identity, London, IB Tauris, 2016.

11 Z. MAASRI, Cosmopolitan Radicalism: The Visual Politics of Beirut's Global Sixties, Cambridge, Cambridge University Press, 2020. 
alleanza dei partiti della destra cristiana nel 1967, ma anche una trasformazione del maronitismo ideologico, che finisce per rivendicare la tesi di una partizione del Libano su base confessionale, accarezzando l'ipotesi di un'entità cristiana sotto forma di cantone o di vero e proprio stato indipendente ${ }^{12}$. L'Université Saint-Esprit de Kaslik, fondata nel 1938 dall'Ordine libanese maronita, è l'incubatrice di un pensiero militante che amalgama ed espande temi dell'isolazionismo maronita, del fenicismo, dell'eccezionalismo libanese (ossia del Libano come terra di rifugio delle minoranze cristiane del Medio Oriente) e li vira in una direzione anti-marxista e anti-islamica ${ }^{13}$, offrendo una copertura ideologica alle tendenze suprematiste cristiane (perlopiù maronite) che convergono nel Fronte libanese ${ }^{14}$. Il tema dell'entità cristiana minacciata dal nazionalismo arabo, dal radicalismo islamico, dal marxismo (o da una combinazione dei tre elementi), unito a slogan e frasi a effetto sulla perseveranza e sulla resistenza contro le minacce di sottomissione e annientamento, è dominante nei discorsi di Bashir al-Jumayyil. Secondo Thuselt ${ }^{15}$, ciò segna una transizione nel discorso politico cristiano libanese dalla percezione di sé come avanguardia della modernizzazione dentro uno stato nazionale definito da caratteristiche e confini risalenti all'antichità alla percezione di sé come minoranza etnica sotto la costante minaccia dell'assimilazione. Questo discorso, come avrò modo di mostrare, riemerge nelle ansie veicolate da esponenti politici cristiani negli ultimi anni.

Non è infine mia intenzione negare la pregnanza del sistema politico confessionalista, definibile come un sistema consociativo di spartizione del potere, delle cariche elettive e istituzionali, e delle maggiori posizioni nella pubblica amministrazione secondo un meccanismo di

12 E. Hagopian, Maronite Hegemony to Maronite Militancy: The Creation and Disintegration of Lebanon, in "Third World Quarterly", n. 4, 1989, pp. 101-117.

${ }^{13}$ A. Henley, Politics of a Church at War: Maronite Catholicism in the Lebanese Civil War, in "Mediterranean Politics", n. 3, 2008, pp. 353-369.

${ }^{14}$ L. SNIDER, The Lebanese Forces: Their Origins and Role in Lebanon's Politics, in "Middle East Journal", n. 1, 1984, pp. 1-33.

G. Hage, Religious Fundamentalism as a Political Strategy: The Evolution of the Lebanese Forces' Religious Discourse During the Lebanese Civil War, in "Critique of Anthropology", n. 1, 1992, pp. 27-45.

15 C. Thuselt, "Dream of a Republic": Lebanese Political Parties as "Real Parties", Roskilde, Roskilde University, 2018, tesi di dottorato. 
quote riservate alle differenti confessioni religiose riconosciute dallo stato, sulla base di una serie di convenzioni costituzionali e di meccanismi informali. La più recente ricerca sul confessionalismo libanese ha analizzato le sue dimensioni spaziali e la sua economia politica, mettendone bene in evidenza la natura strutturalmente clientelare ${ }^{16}$. Nell'inopportunità di soffermarmi ulteriormente su un argomento ben studiato, assumo come note alcune considerazioni di fondo sulla natura complessiva e sulle dinamiche del quadro politico libanese.

\section{Una polarizzazione incompleta: partiti di massa e notabilato clientelare}

La politica libanese dal 2005 a oggi è perlopiù letta attraverso la contrapposizione tra due eterogenee coalizioni, note come "8 marzo" e "14 marzo", grossolanamente definibili rispettivamente come filo-siriana e anti-siriana, e in larga misura riconducibili agli equilibri di forza tra le potenze regionali che vedono nel Libano un'arena di confronto e di misurazione della propria potenza. Alla prima alleanza fanno capo Hezbollah, il movimento Amal, e, nel campo cristiano, il Movimento patriottico libero di Michel Aoun (presidente della repubblica dal 31 ottobre 2016), il Movimento mardaita di Sulayman Franjiyyah e la Federazione rivoluzionaria armena (Tashnag). Alla seconda alleanza appartengono il Movimento del futuro dell'ex primo ministro Sa'd al-Din al-Hariri e, nel campo cristiano, le Forze libanesi di Samir Ja'ja'. Più eclettico rimane il posizionamento del Partito socialista progressista di Walid Junbulat e, nel campo cristiano, del partito delle Falangi libanesi.

Molte ricerche sul campo condotte tra il 2006 e il 2010 hanno registrato un'esasperata polarizzazione, legata alle prolungate dimostrazioni della coalizione "8 marzo" contro il governo di Fu'ad Sanyura e poi agli scontri avvenuti a Beirut il 7 maggio 2008. In occasione delle elezioni parlamentari del 2009 è stata osservata una crescente centralità

16 In particolare, per quanto riguarda la connessione tra la crisi del sistema politico confessionalista e la crisi economica scoppiata nel marzo 2020, oltre che per una panoramica concettuale sulla nozione di confessionalismo, rimando a un mio precedente contributo. Si veda in F. Mazzucotelli, Fragments of Lebanon: Sectarianism and the Financial Crisis, in "Il Politico", n. 1, 2020, pp. 24-42. 
delle "macchine elettorali" (mākīnāt intihăbiyyah) e delle reti clientelari dei partiti di massa, che hanno fagocitato anche le pratiche di mobilitazione politica all'interno delle componenti studentesche universitarie in occasione del rinnovo dei rappresentanti di ateneo ${ }^{17}$, e si sono persino intrecciate con il tifo calcistico legato alle principali squadre di Beirut, le cui specificità storiche e sociali sono state ricondotte nell'alveo di narrative partitiche e confessionali omogeneizzanti ${ }^{18}$.

Uno dei fenomeni più interessanti da osservare e più controversi riguarda l'impatto dell'estesa polarizzazione politica nel periodo compreso tra il 2006 e il 2010 sulle dinamiche clientelari legate al notabilato locale, spesso scevro di connotazioni ideologiche nette e chiaramente discernibili. Al tipo ideale del potere tradizionale sono usualmente ricondotte le pratiche e le figure che fondano la propria legittimazione sui rapporti di parentela (anche attraverso le alleanze matrimoniali e i legami clanici), sul patrimonio personale e familiare, sull'appartenenza comunitaria, con un forte ancoraggio nella dimensione territoriale. Questo tipo di autorità tradizionale è sovente correlata alla coltivazione di relazioni personali dirette, alla munificenza, allo scambio di favori, alla partecipazione agli eventi sociali e soprattutto alla capacità (agita o potenziale) di ricorrere alla forza tramite i propri uomini di fiducia (qabadāy) ${ }^{19}$. A queste forme di potere tradizionale si sarebbero opposte nel diciannovesimo secolo le chiese cattoliche, in particolare quella maronita sotto l'influenza del clero di origine contadina e degli ordini monastici, e nel ventesimo secolo i nuovi capi carismatici legati ai fenomeni di inurbamento e alla politica di massa ${ }^{20}$. A

${ }^{17} \mathrm{~B}$. LEFORT, Un vote de substitution? Mobilisations et pratiques électorales estudiantines. Le cas des élections à l'Université Saint-Joseph, in M. CATusse, K. Karam, O. LAmloum (eds.), "Métamorphoses des figures du leadership au Liban", Beyrouth, Presses de l'IFPO, 2011, pp. 105-134.

18 O. LAMLOUM, Quand la guerre ritualisée est au service de la passion partisane. Football et élections au Liban, in M. CATUSSE, K. KARAM, O. LAMLOUM (dir.), "Métamorphoses des figures du leadership au Liban”, Beyrouth, Presses de l'IFPO, 2011, pp. 135-163.

${ }^{19}$ Il termine colloquiale qabadāay, mutuato dal turco kabadayl, ha una connotazione ambigua di "uomo forte", perlopiù contraddistinto dall'ostentazione di una virilità aggressiva, e indica solitamente coloro che, come i "bravi" manzoniani, si occupano di portare a termine pressioni psicologiche, minacce verbali e ritorsioni fisiche per conto del proprio signore o capo di riferimento.

${ }^{20}$ M. ChaOUL, Zahlé: de la za'âma nationale à la za'âma dépendante, in F. MERMIER, S. Mervin (eds.), Leaders et partisans au Liban, Paris, Karthala, 2012, pp. 435-459. 
partire dagli anni Trenta del ventesimo secolo, i primi partiti di massa con una base ideologica, formati in prevalenza da studenti, professionisti del ceto medio e da lavoratori di recente inurbamento, si pongono come rappresentanti della gente in contrapposizione ai notabili ( $z u$ ' $a m \bar{a}^{\prime}$ ), evidenziando tensioni sociali e di classe interne a ogni comunità confessionale ${ }^{21}$. Lo scoppio della guerra civile, con la creazione di milizie dotate di una direzione politica e militare unitaria, accentua la critica nei confronti delle famiglie tradizionali del notabilato, dipinto come il retaggio di un obsoleto feudalesimo.

Secondo Thuselt22, l'ideologia politica di Bashir al-Jumayyil e del Fronte libanese si basa su due punti cardinali: da un lato, un'idea della nazione come comunità di volontà, con una sua missione o "causa" (qadiyyah) alimentata da una visione teleologica della storia; dall'altro lato, un esplicito posizionamento "popolare" ( $\left.\breve{s}^{\prime} a b \bar{l}\right)$, ma che si potrebbe propriamente definire populista o völkisch, che si contrappone all'elitismo della politica dei notabili e aspira alla formazione di uno stato "forte" ( $q \bar{a} w \bar{\imath})$, paradossalmente proprio nel momento in cui le milizie surrogano le prerogative dello stato ed esibiscono (con più o meno successo) gli attributi tipicamente ascritti alla sovranità statuale.

Bashir al-Jumayyil e le Forze libanesi rivendicano, da un lato, la piena indipendenza del Libano con il suo carattere non arabo e, dall'altro, rigettano la politica delle clientele e dei compromessi tra gli esponenti delle grandi famiglie di notabili, possidenti terrieri e alta borghesia urbana. Il falangismo e ancora di più le milizie cristiane in tempo di guerre sono segnate da un carattere piccolo-borghese e populista, a cui si aggiunge un elemento di giovanilismo maschilista legato al vigore fisico, inteso anche come specchio del vigore morale di una comunità cristiana finalmente liberata dai vincoli del feudalesimo. Il maronitismo militante cerca di proiettare un'immagine di ordine e unanimità legata a un'idea di risveglio e di consapevolezza (wa $\mathfrak{l}$ ) antifeudale ${ }^{23}$.

${ }^{21}$ C. Thuselt, "Dream of a Republic": Lebanese Political Parties as "Real Parties", cit..

${ }^{22}$ C. Thuselt, Milizen als Surrogat eines dysfunktionales Staates: Das Beispiel der "Lebanese Forces", in "Sicherheit und Frieden (S+F) / Security and Peace", n. 4, 2015, pp. 193-199.

${ }^{23}$ M.-C. Aulas, The Socio-Ideological Development of the Maronite Community: The Emergence of the Phalanges and the Lebanese Forces, in "Arab Studies Quarterly", n. 4, 1985, pp. 1-27. 
Scrivendo agli albori della guerra civile, Stoakes ${ }^{24}$ rilevava come il partito delle Falangi libanesi si rivolgesse prevalentemente agli strati sociali più bassi della componente maronita, e in particolare a quelli di recente urbanizzazione nella periferia povera di Beirut, utilizzando il populismo, il culto delle gerarchie e le ostentazioni di virilità come richiami per la gioventù suburbana. Questa tensione sociale tutta interna alle comunità cristiane libanesi, e in particolare a quella maronita $^{25}$, rimane sopita negli anni successivi all'accordo di al-Ta'if, durante i quali l'elemento di discrimine è costituito dal rapporto da tenere nei confronti delle ingerenze del governo siriano in Libano, per poi riemergere a partire dalle elezioni parlamentari del 2000.

Una contrapposizione netta tra forme tradizionali di esercizio del potere e forme modernizzanti, così come tra legittimazione tradizionale e legittimazione carismatica, non appare tuttavia oggigiorno plausibile.

Da un lato, lo studio delle dinamiche nei territori a maggioranza cristiana del Libano del nord mostra come l'emersione di alcune famiglie quali detentrici del potere sia un processo relativamente recente ed eminentemente moderno, legato al sistema di governo del tardo periodo ottomano, più che il retaggio di strutture arcaiche. Si nota così una tendenza alla manipolazione genealogica, tesa a creare un passato familiare idealizzato a partire dalla glorificazione del fondatore, tipicamente presentato come eroe combattente o come santo in linea con la tradizione religiosa locale, invece che come un esattore fiscale per conto del governatore ottomano o come l'intermediario di latifondisti assenti ${ }^{26}$. Dall'altro lato,

${ }^{24}$ F. StoAkes, The Supervigilantes: The Lebanese Kataeb Party as a Builder, Surrogate and Defender of the State, in "Middle Eastern Studies", n. 3, 1975, pp. 215-236.

25 In larga misura differente è il caso della comunità greco-ortodossa, caratterizzata in parte da una plurisecolare presenza urbana e da una relazione stretta con l'identità araba e siriana, che ha anche ripercussioni in campo politico. Ancora diverso è il caso della comunità armena, in particolare la parte maggioritaria legata alla Chiesa apostolica, ove è presente un elemento di differenziazione etnico-linguistica e una contrapposizione interna tra partiti armeni relativa all'identità diasporica e, in passato, al rapporto con l'Armenia sovietica. Si veda F. MAZzuCotelli, Armenian Clergy and Conflict Management in Lebanon, 1920-1994, in C. FrapPI, P. Sorbello (a cura), "Armenia, Caucaso e Asia Centrale. Ricerche 2020”, Venezia, Edizioni Ca’ Foscari, 2020, pp. 139-160.

${ }^{26}$ C. DOUAYHI, Réflexions autour de la za'âma maronite au Liban-Nord, in F. MERmier, S. Mervin (dir.), "Leaders et partisans au Liban", Paris, Karthala, 2012, pp. 481-492. 
sia in ambito urbanistico ${ }^{27}$ sia in ambito di sociologia politica ${ }^{28}$ è stata messa in luce l'estrema porosità del confine tra dimensione urbana e dimensione rurale, che si riflette nella porosità del confine tra modalità tradizionali, moderne e carismatiche di gestione del potere.

Studiando in periodi diversi gli esponenti politici di tipo clientelare e notabilare della città di Zahlah, Hottinger ${ }^{29}$ e Gubser ${ }^{30}$ notavano già prima della guerra civile come gli esponenti del notabilato mirassero ad assicurare la distribuzione di risorse o impieghi pubblici ai propri seguaci in cambio di indefessa lealtà, e di come il meccanismo clientelare prevalesse sulle prese di posizione su singoli temi o sulla coerenza ideologica. Le vicende politiche ed elettorali già allora evidenziavano non solo il carattere aleatorio e strumentale delle alleanze e degli allineamenti politici, ma anche la turbolenza della politica clientelare, costretta continuamente ad adeguarsi ai cambiamenti indotti dalla situazione politica nazionale (libanese) e regionale (mediorientale), e pertanto trovandosi spesso nella condizione di doversi agganciare a qualche figura di caratura nazionale o persino a un governo estero.

Le vicende delle tre generazioni di politici della famiglia Skaf a Zahlah, studiate da Chaoul31, o quelle delle "famiglie conosciute" ('ā'ilāt ma 'rüfah) del Libano del nord, analizzate da Douayhi², illustrano bene la difficoltà di inquadrare in maniera dicotomica la relazione tra notabilato di tipo "tradizionale" e partiti politici di massa.

Secondo Catusse e Mouawad ${ }^{33}$, ad esempio, l'anatomia dei risultati

27 J.-L. Arnaud, Trop grand Beyrouth?, in J.-L. Arnaud (dir.), “ Beyrouth, Grand Beyrouth”, Beyrouth, Presses de l'IFPO, 1996, pp. 209-227. C. EDDÉ, Beyrouth, le MontLiban et ses environs au XIXe siècle: pratiques de l'espace. Le cas de la communauté maronite, in J.-L. ARnAUd (ed.), “ Beyrouth, Grand Beyrouth”, Beyrouth, Presses de l'IFPO, 1996, pp. 35-49.

${ }^{28}$ I. RIVOAL, Intimité, mise en scène et distance dans la relation politique au Liban, in F. Mermier, S. Mervin (dir.), "Leaders et partisans au Liban”, Paris, Karthala, 2012, pp. $137-163$.

${ }^{29}$ A. Hottinger, Zu'amā' and Parties in the Lebanese Crisis of 1958, in "Middle East Journal”, n. 2, 1961, pp. 127-140.

${ }^{30}$ P. GubSER, The Zu'amā' of Zahlah: The Current Situation in a Lebanese Town, in "Middle East Journal", n. 2, 1973, pp. 173-189.

${ }^{31}$ M. Chaoul, Zahlé: de la za'âma nationale à la za'âma dépendante, cit..

32 C. DouAYHI, Réflexions autour de la za'âma maronite au Liban-Nord, cit..

${ }^{33}$ M. Catusse, J. Mouawad, "Les choix de Jbeil". Notabilité locale, mobilisations nationales et réalignements, in M. CATusse, K. Karam, O. LAmloum (dir.), "Métamorphoses des figures du leadership au Liban”, Beyrouth, Presses de 1'IFPO, 2011, pp. 33-77. 
delle elezioni parlamentari del 2009 nel collegio di Jbeil ${ }^{34}$ mostra una metamorfosi del notabilato locale e il relativo declino della capacità delle grandi famiglie del territorio di mobilitare sufficienti risorse economiche e simboliche per fronteggiare la polarizzazione del quadro politico. Nonostante le variabili strettamente locali e persino campanilistiche continuino a giocare un ruolo di rilevante importanza nella selezione delle candidature e nei comportamenti di voto, l'analisi dei risultati del 2009 sembrerebbe evidenziare il successo delle strutture partitiche e un allineamento delle affiliazioni politiche in funzione del quadro nazionale libanese e delle identità confessionali, esacerbate $\mathrm{e}$ strumentalizzate dai partiti organizzati (Movimento patriottico libero, Forze libanesi, Falangi libanesi) per accreditarsi quali campioni della propria comunità. I patrimoni politici delle famiglie che hanno tradizionalmente monopolizzato la rappresentanza politica locale verrebbero, secondo questa lettura, vieppiù ancorati agli antagonismi partitici a livello nazionale, secondo relazioni a geometria variabile che si intersecano con le identità confessionali, le frizioni sociali e le dinamiche generazionali. Questi cambiamenti raramente mettono in discussione le pratiche di creazione e mantenimento del consenso attraverso la fornitura selettiva di risorse o servizi altrimenti non accessibili o non facilmente disponibili per la popolazione. Anche i partiti strutturati, secondo Catusse e Mouawad, ricorrono alle cosiddette "chiavi" (mafātīh ), ossia luogotenenti in grado di raccogliere rimostranze e richieste del territorio e di trasmetterle ai candidati, organizzando in cambio il consenso elettorale.

Dentro questa cornice, ritengo molto meritevoli di attenzione i riallineamenti che si sono prodotti in occasione delle elezioni locali del 2016 e poi delle elezioni legislative del 2018. Seppur senza potermi dilungare nei dettagli in questa sede, e quindi trovandomi ad asserire più che a dimostrare, la linea di tendenza generale che emerge dalle due consultazioni vede i due principali partiti cristiani di massa

\footnotetext{
${ }^{34}$ In quella occasione si votava con un diverso sistema elettorale, sempre basato sulla ripartizione dei seggi in palio su base confessionale e territoriale, ma con un meccanismo maggioritario all'interno di ogni distretto amministrativo. La riforma del sistema elettorale è stata uno dei cavalli di battaglia dei partiti cristiani e soprattutto, per molti anni, dell'ex patriarca maronita Nasrallah Butrus Sfayr, secondo cui il sistema varato nel 1992 non era adeguatamente rappresentativo.
} 
(ossia il Movimento patriottico libero e le Forze libanesi) intensificare accentuatamente la loro retorica contro il potere dei notabili e delle famiglie tradizionali, definite come feudali e obsolete. Il tentativo da parte di queste ultime di difendersi, descrivendo i candidati dei partiti nazionali come agitatori estranei al contesto locale del villaggio o del quartiere, ha avuto esiti modesti: come già nel 2009, si sono salvati quei notabili ed esponenti politici di tipo "tradizionale" in grado di agganciarsi alle "macchine elettorali" dei partiti nazionali35. Paradossalmente, l'introduzione di un sistema proporzionale con voto di preferenza, sebbene complicato dal meccanismo di quote riservate su base confessionale, appare aver diminuito il peso e l'influenza degli esponenti della politica notabilare $\left(z a^{\prime} a \bar{a} m a h\right)$, molti dei quali non sono stati rieletti.

Le due campagne elettorali del 2016 e del 2018 sono state segnate da un'intensa competizione intra-cristiana, che tuttavia non si è giocata solo sul piano delle affiliazioni nazionali (tra "8 marzo" e "14 marzo", come era stato nel 2009) e internazionali, ma anche (e forse soprattutto) su una competizione del tutto interna alla sfera pubblica cristiana, nella quale i partiti di massa hanno utilizzato le carte del populismo e della difesa degli interessi comunitari/confessionali in funzione antinotabilare ${ }^{36}$. Questa situazione non è scevra da contraddizioni: le Forze libanesi, per esempio, si presentano oggi sotto il doppio lato della "modernità" e della "tradizione", da un lato esibendo un'ideologia nazionalista di tipo moderno in contrapposizione al sistema oligarchico e clientelare delle grandi famiglie feudali cristiane, dall'altro richiamandosi fermamente ai valori e alla dottrina

35 A. El-Hage, Enjeu politique ou purement local? Tannourine s'exprimera dimanche, in "L'Orient-Le Jour", 25 maggio 2016, https://www.lorientlejour.com/article/987775/enjeupolitique-ou-purement-local-tannourine-sexprimera-dimanche.html (ultimo accesso: 30 agosto 2021), O. ANTAR, La bataille à Zghorta prende une nouvelle tournure, in "L'Orient-Le Jour", 25 maggio 2016, https://www.lorientlejour.com/article/987785/la-bataille-a-zghortaprend-une-nouvelle-tournure.html (ultimo accesso: 30 agosto 2021). A. EL-HAGE, Anatomie du vote à Zahlé, in “L'Orient-Le Jour", 12 maggio 2018, https://www.lorientlejour.com/article/1115076/anatomie-du-vote-a-zahle.html (ultimo accesso: 30 agosto 2021). Y. ABI AKL, Anatomie du vote à Kesrouan-Jbeil, in "L'Orient-Le Jour", 19 maggio 2018, https://www.lorientlejour.com/article/1116442/anatomie-du-vote-a-kesrouan-jbeil.html (ultimo accesso: 30 agosto 2021).

36 Questa dinamica è stata oltremodo evidente sulle piattaforme di comunicazione sociale e sui canali televisivi libanesi. 
della Chiesa maronita, della quale ambiscono spesso a presentarsi come i rappresentanti temporali ${ }^{37}$.

\section{Il capo politico: eroe, figlio del popolo, specchio della comunità}

È ben vero che i partiti politici libanesi sono perlopiù apparati elettorali ideologicamente gelatinosi che ruotano intorno al culto della personalità del capo. La legittimazione carismatica di quest'ultimo si configura come una relazione tra il capo e i suoi seguaci che si basa sulla difesa di una causa e sul desiderio di costituire una comunità affettiva. Il capo, con le sue res gestae, non è tanto un individuo eccezionale per le sue qualità o per i risultati effettivamente conseguiti, quanto colui che incarna il progetto politico della comunità di appartenenza. È per questa ragione che l'agiografia del capo è sovente declinata attraverso la semantica del "sogno" ( $\mathrm{hulm})$, in contrapposizione alla deprimente realtà $\mathrm{o}$ alla disfunzionalità del sistema politico ed economico, o attraverso un registro religioso, che riprende esplicitamente i temi della passione (di Cristo) e della palingenesi, perlopiù equiparata alla rivivificazione dei valori cristiani e degli stili di vita associati a una raffigurazione idealizzata della vita rurale nello stereotipo del villaggio di montagna ${ }^{38}$.

In ambito cristiano, il processo di iconizzazione del capo politico attraverso un simbolismo religioso desunto largamente dalla tradizione maronita si rivela in tutta la sua pregnanza nella figura di Bashir al-Jumayyil. L'agiografia e la "canonizzazione" dei capi politici sono largamente attestate, in contesti diversi e anche all'interno di regimi formalmente laici, nel paesaggio politico mediorientale. Le "icone" del capo e il suo stesso corpo servono a veicolare emozioni, memorie e senso di appartenenza a una comunità politica. La particolarità della costruzione del mito e dell'icona di Bashir al-Jumayyil risiede, secondo Haugbølle ${ }^{39}$, nell'ubiquità

${ }^{37}$ E. Aubin-Boltanski, Samir Geagea: le guerrier, le martyr et le za'îm, in F. MerMIER, S. MERVIN (dir.), "Leaders et partisans au Liban" Paris, Karthala, 2012, pp. 57-80.

${ }^{38}$ Z. MAASRI, Off the Wall: Political Posters of the Lebanese Civil War, London, IB. Tauris, 2009, pp. 111-113. Si veda anche in C. ThuseLt, Milizen als Surrogat eines dysfunktionales Staates, cit.

${ }^{39}$ S. HaUgbøLle, The Secular Saint. Iconography and Ideology in the Cult of Bashir Jumayil, cit.. 
dei riferimenti alla croce e al cedro (simbolo saturo di associazioni con l'Antico Testamento) che permettono alla propaganda falangista di raffigurarlo quale prototipo della vittima sacrificale. Una pletora di materiali audiovisivi, i cui modi di fruizione sono importanti tanto quanto i contenuti, alimentano una venerazione densa di riferimenti espliciti e di sottotesti impliciti di tipo religioso: la morte del capo riecheggia la passione di Cristo e la passione del Libano, e per converso far rivivere politicamente lo spirito del capo vuol dire vivere in maniera assertiva la propria identità libanese e cristiana.

Le pratiche di commemorazione dei capi politici cristiani recuperano molti elementi di quello che si potrebbe definire come l'archivio simbolico del cristianesimo libanese, a cominciare dalle figure dei due santi combattenti, san Giorgio e sant'Elia, che assumono il ruolo di simboli nazionalisti libanesi e di protettori contro i nemici e gli invasori stranieri. Anche nell'iconografia contemporanea di Samir Ja'ja', studiata attentamente da Aubin-Boltanski ${ }^{40}$, appaiono evidenti un misticismo radicale dagli aspetti messianici e una retorica impregnata di nozioni di missione e martirio. Capo miliziano violento e devotissimo cristiano, Samir Ja'ja' riprende scientemente un'antropologia della santità che, nelle tradizioni della cristianità orientale, alterna i percorsi della testimonianza combattente (i santi guerrieri) e dell'ascetismo monacale. Ja'ja', che agli esordi della sua parabola militante ama definirsi un pecoraio e un montanaro, per segnare il suo distacco sia dalle grandi famiglie dell'area di origine della sua famiglia sia dagli esponenti politici di estrazione borghese urbana, viene sovente messo in correlazione con la figura di Taniyus Shahin, mistico e capopopolo, istigatore della rivolta contadina antifeudale del 1858, ma anche con il santo della montagna Charbel Makhluf, che a partire dagli anni Cinquanta del ventesimo secolo viene esaltato come la quintessenza del maronitismo, riunendo in sé ascetismo da anacoreta e amore viscerale per la propria terra.

La costruzione dell'agiografia di san Charbel Makhluf, secondo Heyberger ${ }^{41}$, è assai importante per comprendere le tensioni che attraverso il cristianesimo maronita. Il culto del santo appare debitore di una religiosità tipica del clericalismo controrivoluzionario e rurale della

\footnotetext{
40 E. Aubin-Boltanski, Samir Geagea: le guerrier, le martyr et le za'îm, cit..

${ }^{41}$ B. Heyberger, Saint Charbel Makhlouf, ou la consécration de l'identité maronite, cit..
} 
Francia ottocentesca, e si alimenta fortemente dell'ideologia nazionalista di Maurice Barrès, incentrata su la terre et les morts. La mitologia del martirio trova un parallelismo nella storia del popolo maronita, immaginato come sempre a rischio di assimilazione o di sottomissione di fronte agli invasori musulmani. La retorica della terra e delle radici, d'altro canto, rafforza un'idea di Libano quale entità cristiana nella quale si radica la storia del popolo maronita, che gode di un rapporto privilegiato con la terra e pertanto di una rivendicazione di legittimazione politica superiore a quella di altre confessioni religiose. Nel culto del santo emergerebbe così un legame inscindibile tra religione, territorio e identità libanese, in cui i cristiani maroniti assurgerebbero al ruolo di guardiani del paese e della sua missione di baluardo del cristianesimo in Medio Oriente.

La compenetrazione di elementi carismatici, populisti e messianici traspare con evidenza anche dalle interviste realizzate da Lefort ${ }^{42}$ a un campione di militanti e sostenitori di Michel Aoun, allora capo del Movimento patriottico libero e non ancora divenuto presidente della repubblica. Egli emerge dai ricordi dei seguaci come uomo provvidenziale ed eroe popolare che lotta per la restaurazione di uno stato sovrano fondato sul monopolio della violenza legittima a fronte dei gruppi armati miliziani e sull'indipendenza nazionale a fronte delle ingerenze straniere. Dalle "memorie vive" dei militanti emerge in particolare l'epopea tragica della guerra di liberazione condotta tra il 14 marzo 1989 e il 13 ottobre 1990 contro le milizie delle Forze libanesi e contro l'esercito siriano. Affiorano in alcuni racconti i temi della morte e della rinascita, che inducono persino ad accostare la parabola di Aoun al mito di Tammuz/Adonis o, ancora una volta, alla passione di Cristo. Al capo politico, la cui missione è quella di far transitare la propria comunità verso il futuro, molti seguaci attribuiscono un dono quasi profetico di saper leggere le linee di tendenza storiche e politiche, e pertanto di anticipare le scelte strategiche che possano offrire una migliore rappresentanza politica ai cristiani libanesi. La natura di "salvatore" (muhalliṣ) attribuita da alcuni sostenitori a Michel Aoun risiede proprio nella speranza di una rinnovata centralità dei cristiani a fronte

42 B. LEFORT, Répresentations du leadership et memoires vives chez les militants aounistes, in F. Mermier, S. Mervin (eds.), "Leaders et partisans au Liban”, Paris, Karthala, 2012, pp. 219-262. 
dei pericoli (veri o presunti) di riduzione in minoranza all'interno di un ambiente arabo-musulmano considerato spesso come minaccioso. L'insistenza sulla preservazione dell'identità e delle tradizioni libanesi, soprattutto di fronte all'ipotesi di un definitivo impianto dei profughi palestinesi, ripropone la sovrapposizione tra la difesa dello stato nazionale e la difesa dell'identità cristiana. Al di là della retorica anticonfessionalista, inoltre, le posture sovraniste e le aspre critiche al sistema scaturito dall'accordo di al-Ta' if si fondano in realtà sulla convinzione che l'assetto postbellico sia nettamente penalizzante per i cristiani, e che l'erosione delle prerogative di organi tradizionalmente attribuiti a esponenti cristiani si traduca in una crescente marginalizzazione e vulnerabilità. Si coglie in molte testimonianze un afflato quasi messianico, nel quale il capo funge da protettore e da guida a fronte delle minacce dei gruppi jihadisti e della paura di essere relegati in una posizione fortemente subalterna. In questo modo, benché ufficialmente non confessionalista, il discorso prevalente tra molti sostenitori ed elettori di Aoun ha tratti marcatamente confessionali.

La narrazione di Aoun e del Movimento patriottico libero contrappone l'ideale unionista di uno stato "forte" $(q \bar{a} w \bar{l})$, garantito dalle forze armate, all'ordine miliziano e alle ipotesi di partizione federale che creerebbero una ridotta cristiana debole ed esposta ai ricatti dei propri protettori stranieri. Nella rivendicazione del ruolo di vero rappresentante della "strada" maronita, sembra affiorare il paradigma ottocentesco della contrapposizione tra famiglie feudali, patriarcato e 'ámmiyyāt (sollevazioni rurali), ancora una volta riproponendo il parallelo con la figura di Taniyus Shahin e con i moti del 1858.

D'altro canto, secondo Abirached ${ }^{43}$, la vicenda politica di Aoun mostra come la relazione di tipo carismatico possa iscriversi in una lunga durata, affrancandosi da nozioni di vittoria e sconfitta basate su una temporalità di breve periodo, e in generale di come il fenomeno carismatico libanese si presti a una problematizzazione delle letture eccessivamente unilaterali, mostrando al contrario la complessità delle relazioni tra il capo e i suoi sostenitori. Il fenomeno carismatico si spiega non tanto per le caratteristiche personali o i risultati obiettiva-

43 P. ABIRACHED, Charisme, pouvoir et communauté politique: la figure de Michel Aoun, in F. Mermier, S. Mervin (eds.), "Leaders et partisans au Liban", Paris, Karthala, 2012, pp. 33-55. 
mente conseguiti dal capo politico, bensì per la capacità di quest'ultimo di attivare (o riattivare) frontiere politiche e identitarie che permettano ai membri del gruppo di riconoscersi come soggetto politico collettivo. Secondo l'analisi di Abirached, è la costruzione di una prossimità emotiva immaginata a rianimare costantemente la relazione con il capo politico e a permettere di proiettare le proprie ansie e aspettative individuali all'interno di una dimensione interpersonale e comunitaria, creando insomma quella "comunanza emotiva" che Weber ravvisa come elemento centrale della dominazione carismatica ${ }^{44}$.

La persistenza del fenomeno carismatico e la sua riproduzione durante il periodo dell'esilio, dal 1991 al 2005, indica come il radicamento della relazione bilaterale tra capo e seguaci prevalga sull'efficacia complessiva del progetto politico nella definizione del fenomeno del carisma nella politica libanese. Il rientro di Aoun in Libano induce in ogni caso a incardinare la guida carismatica all'interno di una organizzazione politica strutturata sotto forma di partito. La nuova identità politica viene rinvenuta nella lotta al clientelismo e alla corruzione delle oligarchie tradizionali. Il confessionalismo vigente viene additato come l'intersezione tra il feudalesimo rappresentato dalle grandi famiglie legate alla proprietà fondiaria, i signori della guerra confessionale riconvertiti in capi di partito, e una oligarchia di affaristi e speculatori finanziari.

Nel rivendicare la natura di movimento nazionale e nazionalista che mira a ricostruire uno stato forte al posto dello "staterello" (duwaylah) esistente, il movimento di Aoun si presenta sin dagli albori come un progetto che mira a difendere gli interessi e i diritti della popolazione cristiana ${ }^{45}$. A differenza delle Forze libanesi, che a lungo accarezzano l'idea di un cantone cristiano e di una segregazione territoriale su base confessionale, il movimento di Aoun ritiene che solo una strategia di difesa promossa e realizzata dallo stato sulla totalità del suo territorio possa efficacemente garantire la popolazione libanese, e in particolare quella cristiana. Il discorso politico del movimento aounista alterna così l'argomento della sovranità (siyādah) nazionale al registro

\footnotetext{
${ }^{44} \mathrm{H}$. BRUHNS, Le charisme en politique: idée seduisante ou concept pertinent?, in "Cahiers du Centre de recherches historiques", n. 24, 2000.

${ }^{45} \mathrm{P}$. ABIRACHED, Charisme, pouvoir et communauté politique: la figure de Michel Aoun, cit..
} 
identitario e confessionale cristiano, come si vede bene nel "Documento delle proposizioni cristiane libanesi" del dicembre 200746.

Oltre alla rivendicazione immediata di un riequilibrio dei poteri istituzionali, con una "riabilitazione" delle prerogative della presidenza della repubblica e la richiesta di una maggiore rappresentanza dei cristiani all'interno del governo, della pubblica amministrazione, e degli apparati di sicurezza, il testo è di grande rilevanza per tre motivi. In primo luogo, il documento definisce il patriarcato maronita come autorità morale e "autorità religiosa nazionale incontestata" che deve vegliare sull'applicazione delle regole della vita democratica all'interno della componente cristiana della società libanese, pur non dovendo intromettersi direttamente nelle scelte immediatamente politiche. ${ }^{47}$ In secondo luogo, il documento sostiene la necessità di rispettare il principio della democrazia competitiva all'interno di ciascuna comunità confessionale e il principio della democrazia consensuale (tawāfuqiyyah) a livello nazionale. In terzo luogo, il documento indica quelli che, secondo i suoi estensori, sarebbero i pericoli immediati che minaccerebbero il Libano e la sua popolazione cri-

\footnotetext{
${ }^{46} \mathrm{Si}$ veda in: https://mplbelgique.wordpress.com/2007/12/06/document-des-propositions-chretiennes-libanaises-2/ (ultimo accesso: 30 agosto 2021).

${ }^{47}$ In realtà, la relazione tra le gerarchie ecclesiali maronite e le istituzioni pubbliche dello stato libanese è stata fortemente influenzata dalla percezione da parte della Chiesa degli interessi politici dei cristiani libanesi, mettendo frequentemente il patriarcato e la conferenza episcopale maronita in rotta di collisione con la classe politica cristiana riguardo alle priorità e ai mezzi più efficaci per garantire la tutela della popolazione cristiana. Relazioni burrascose tra il patriarcato maronita e la presidenza della repubblica si sono viste con i patriarchi Antuniyus Aridah (1932-1955) e Bulus Butrus al-Ma'ushi (1955-1975), ma soprattutto con il patriarca Nasrallah Butrus Sfayr (1986-2011), che è emerso come rappresentante della comunità maronita, vero e proprio etnarca, nel clima di rassegnazione seguito all'accordo di al-Ta'if. Sfayr ha contestato la classe politica filosiriana e si è posto come garante della restaurazione della piena indipendenza libanese, considerata come condizione vitale per la preservazione dell'entità maronita, vedendo al contrario nella tutela siriana la radice di tutti i mali del paese. Contrariamente agli auspici del "Documento delle proposizioni cristiane libanesi", Sfayr è intervenuto in maniera diretta nelle elezioni del 2009, sostenendo la coalizione "14 marzo". Si veda in S. BAROUdi, P. TABAR, Spiritual Authority versus Secular Authority: Relations between the Maronite Church and the State in Postwar Lebanon: 1990-2005, in "Middle East Critique”, n. 3, 2009, pp. 195-230. Segnalo inoltre N. NASSIF, al-Arğwānī. Bkirkī: hatmiyyah al-dawr am 'ib' al-hiyārr? [Le violet. Le patriarcat maronite: rôle incontournable ou choix difficile?] in M. Catusse, K. Karam, O. Lamloum (dir.), "Métamorphoses des figures du leadership au Liban”, Beyrouth, Presses de l'IFPO, 2011, pp. 203-262.
} 
stiana: il pericolo della ricollocazione definitiva dei profughi palestinesi, che cambierebbe i dati demografici e altererebbe un già precario equilibrio di forza tra cristiani e musulmani, e il pericolo del surplus $d$ 'argent che si concretizzerebbe nell'acquisto massiccio di proprietà fondiarie e immobili. È fondamentale chiarire che il documento non deplora la selvaggia speculazione edilizia che ha deturpato mostruosamente il paesaggio libanese, ma l'acquisto di terreni e case da parte di acquirenti musulmani in territori tradizionalmente a maggioranza cristiana. Nonostante i ripetuti passaggi relativi alla convivenza e al superamento del sistema confessionalista, le proposizioni libanesi del 2007 riaffermano invece la rivendicazione di un ancoraggio territoriale e il desiderio di una segregazione spaziale definita su base religiosa.

\section{La demarcazione territoriale e il posto dell'altro}

I processi di omogeneizzazione confessionale e gli spostamenti forzati di popolazione nel corso della guerra civile e del dopoguerra sono stati ampiamente studiati dalla letteratura esistente, sia sotto il profilo strettamente urbanistico sia sotto un profilo più ampiamente sociologico ${ }^{48}$. Numerosi progetti immobiliari, come quelli del Fondo sociale maronita, sono espressamente riservati a futuri acquirenti cristiani, contribuendo ulteriormente alla segregazione spaziale ${ }^{49}$. Il dibattito intorno alla natura del confessionalismo libanese ne ha messo in luce la natura profondamente spazializzata e il tentativo costante di trasformare gli spazi pubblici urbani in spazi demarcati in senso identitario.

Il lavoro di campo di Mazaeff 50 , condotto nell'area periurbana di 'Ayn al-Rummanah, alle porte di Beirut, mostra la profonda commistione tra la significazione del territorio e la costruzione di una retorica

48 Per una ricognizione di alcune opere principali rimando a un mio contributo sugli spazi pubblici nel centro di Beirut. Si veda in F. Mazzucotelli, La Piazza dei Martiri di Beirut: l'isola che non c'è, in "Storia Urbana", n. 139, 2013, pp. 95-133.

${ }^{49} \mathrm{M}$. FeLSCH, The Rise of Christian Nationalism in Lebanon, cit..

${ }^{50} \mathrm{C}$. MAZAEFF, L'action des Forces Libanaises à Ä̈n al-Remmané: un intense travail de réhabilitation et de socialisation politique, in F. MERMier, S. MERvin (dir.), "Leaders et partisans au Liban", Paris, Karthala, 2012, pp. 263-283. 
fortemente identitaria e confessionale. In questo quartiere di periferia sottoproletaria, definita dalla stigmatizzazione dei suoi giovani come za 'rān (un termine colloquiale dispregiativo traducibile come "borgatari" o "bulli di periferia"), il musulmano viene dipinto come integralmente altro da sé, irriducibilmente alieno e minaccioso, un nemico ontologico nel quadro di una visione semplicistica e spesso ossessiva della storia, della politica locale e delle relazioni tra confessioni religiose. Lo spazio del quartiere diviene la prima linea di questa contrapposizione, un territorio latore del messaggio di barriera simbolica sulla base di visioni essenzializzanti e vittimistiche dell'identità cristiana. La mobilitazione politica fissa l'identità del quartiere e dà a quest'ultimo una missione di testimonianza aggressiva e militante che porta a confessionalizzare lo spazio urbano e di fatto a sottrargli la sua natura pubblica e plurale. L'appropriazione del territorio come spazio egemonizzato dal partito dominante traspare anche dalla diffusione di attività e servizi di carattere assistenziale che in realtà rendono evidente la fragilità dello stato e ne mettono in discussione la capacità di assicurare ai propri cittadini le funzioni e i servizi di base ${ }^{51}$. I partiti cristiani, così come tutti gli altri partiti confessionalisti libanesi, approfittano insomma della debolezza delle istituzioni dello stato per offrire servizi sociali, assistenziali, educativi e sanitari che, da un lato, rafforzano logiche clientelari e, dall'altro lato, rafforzano una dimensione comunitaria angusta che esaspera la nozione di entre-soi, peraltro con il rischio di pulsioni autoritarie.

Come nota Felsch ${ }^{52}$, l'idea di un territorio cristiano geograficamente e storicamente determinato si traduce oggi nell'imperativo di preservarne il carattere confessionale e impedire che vi si insedino troppi musulmani e troppi immigrati africani e sud-asiatici. In alcune aree particolarmente sensibili in quanto énclaves o frontiere confessionali (Jazzin, al-Qubayyat, al-Hadath), le transazioni fondiarie sono di-

${ }^{51}$ Queste considerazioni sono evidentemente estendibili al ruolo egemonico che Hezbollah svolge nell'area della periferia meridionale di Beirut, che ho preso in considerazione nella mia ricerca di dottorato. Sul ruolo dei partiti nel corso della pandemia di Covid-19 e sul loro tentativo di strumentalizzare l'emergenza sanitaria per riasserire il controllo del territorio, rimando a F. MAzzUCOTELLI, Fragments of Lebanon: Sectarianism and the Financial Crisis, cit..

52 M. FelsCH, The Rise of Christian Nationalism in Lebanon, cit.. 
venute fonte di grandissima tensione, alimentata dal timore di un ribaltamento degli equilibri demografici. Alcuni politici cristiani, come l'ex ministro del lavoro e candidato presidenziale Butrus Harb, hanno proposto disegni di legge che avrebbero vietato la compravendita di terre e immobili tra soggetti privati di religione diversa ${ }^{53}$. Le amministrazioni municipali intervengono in deroga alla legislazione vigente per rendere particolarmente difficile l'acquisizione e la registrazione catastale di proprietà fondiarie da parte di acquirenti musulmani ${ }^{54}$. Dietro alla retorica sul mantenimento della concordia sociale, si coglie l'idea che esista un piano (alternativamente sunnita o sciita) di espansione territoriale e assimilazione culturale attraverso l'acquisto di terreni e case.

Le ansie relative agli equilibri demografici stanno alla base anche della posizione intransigente dei partiti cristiani nei confronti delle proposte di modifica della legge sulla cittadinanza (in particolare, sul fatto che una donna libanese sposata con un uomo non-libanese possa o meno trasmettere ai suoi figli la cittadinanza libanese) e sul voto della diaspora libanese.

La guerra civile in Siria ha rafforzato le posture xenofobe e il complesso di accerchiamento, riflesso anche nella creazione di ronde di vigilanti, nell'imposizione del coprifuoco notturno per gli immigrati africani e asiatici e per i rifugiati siriani, negli ostacoli frapposti all'insediamento di questi ultimi e in ultimo alle ripetute pressioni per il loro rimpatrio 55 .

${ }^{53}$ M. MIKDASHI, Lebanese Ideology and Boutros Harb: Separate, But [Kind of] Equal, in "Jadaliyya", 8 gennaio 2011, https://www.jadaliyya.com/Details/23615 (ultimo accesso: 30 agosto 2021).

54 S. HourI, Hassan calls Hadath Muslim property ban unconstitutional, in "The Daily Star", 29 giugno 2019, http://www.dailystar.com.lb/News/Lebanon-News/2019/Jun21/485776-hassan-calls-hadath-muslim-property-ban-unconstitutional.ashx (ultimo accesso: 30 agosto 2021).

55 B. Mroue, Lebanese town bans Muslims from buying, renting property, in "Associated Press", 26 giugno 2019, https://apnews.com/article/media-ap-top-news-international-news-lebanon-lifestyle-ab2518df6f074c3bae9494638840d26a (ultimo accesso: 30 agosto 2021). M. MadorÉ, The Peaceful Settlement of Syrian Refugees in the Eastern suburbs of Beirut: Understanding the causes of social stability, in "Civil Society Knowledge Centre", 1 marzo 2016, https://civilsociety-centre.org/paper/peaceful-settlementsyrian-refugees-eastern-suburbs-beirut-understanding-causes-social (ultimo accesso: 30 agosto 2021). 
La retorica dei "diritti cristiani" appare spesso uno strumento per gli interessi di singoli esponenti politici o dei loro partiti, anche al prezzo di esacerbare le ostilità confessionali. La minaccia esterna rimane un efficace mezzo per mobilitare il consenso per ragioni interne e persino in funzione delle rivalità intra-cristiane ${ }^{56}$.

Più che la formazione di uno spazio di sicurezza comunitario, il nazionalismo cristiano continua a enfatizzare la funzione dello stato libanese in quanto propedeutico alla difesa degli interessi cristiani.

\section{Conclusioni}

Per quanto tatticamente schierati su fronti avversi, che rispondono a diversi allineamenti dal punto di vista della politica regionale e degli equilibri di forza in Medio Oriente, i principali soggetti politici cristiani libanesi evidenziano una sostanziale convergenza di vedute, legate all'obiettivo di preservare lo stato libanese dalle minacce esterne (perlopiù identificate nei gruppi armati di matrice islamista) e interne (identificate in tutto ciò che può mettere in discussione un'identità confessionale monolitica e il sistema politico confessionalista). In questo senso considero emblematica la parabola politica di Jibran Bassil, attuale presidente del Movimento patriottico libero, genero dell'attuale presidente della repubblica Michel Aoun, nonché ministro degli esteri tra il 2014 e il 2020. La sua ambizione neanche troppo nascosta è quella di accreditarsi come il principale esponente politico cristiano, per coltivare la speranza di accedere in futuro alla presidenza della repubblica. Al posto del populismo "anti-casta" del suocero, Bassil ha accarezzato le pulsioni identitarie e le paure dell'elettorato cristiano, giungendo a mettere in discussione la riconciliazione tra drusi e cristiani che l'ex patriarca maronita Sfayr aveva negoziato nel 2000 in funzione antisiriana. La polemica montata nell'agosto del 2019 intorno a un concerto del gruppo musicale Mashrou' Leila, noto per le critiche al maschilismo e all'omofobia, ha visto l'ostentazione di simboli religiosi e la denuncia di un presunto complotto globalista che, attraverso la cosiddetta propaganda omosessuale, avrebbe avuto l'obiettivo di minare l'identità

\footnotetext{
${ }^{56}$ M. FelsCH, The Rise of Christian Nationalism in Lebanon, cit..
} 
cristiana libanese. La virulenta offensiva, nata anche da molto venali interessi materiali e politici legati al controllo del festival internazionale di Byblos, è stata in fondo un pretesto per attaccare i settori laici e progressisti della società libanese che, seppur tra molti limiti, si sono affacciati sulla scena nelle elezioni del 2018. L'utilizzo di una retorica religiosa cristiana diretta non contro i musulmani, bensì contro la minoranza laica e progressista, non costituisce un'anomalia, ma una continuità della logica omogeneizzante del confessionalismo libanese, che di fatto crea una serie di cantoni controllati da un partito egemone.

In un paese dove si moltiplicano gli episodi di aggressione contro le persone migranti, in particolare di origine africana, i partiti cristiani difendono le amministrazioni municipali che, nella vaghezza del quadro giuridico e politico complessivo, hanno adottato regolamenti draconiani di limitazione della libertà degli immigrati e dei rifugiati siriani in nome del "decoro" e della sicurezza. Nel frattempo, mediante il paravento della regolarizzazione del lavoro in nero, le autorità libanesi hanno emanato in questi anni una serie di misure restrittive che di fatto ostacolano i profughi palestinesi e i rifugiati siriani, accusati di "rubare il lavoro" ai cittadini libanesi e di spingere i salari al ribasso 57.

In un clima di perdurante instabilità interna e regionale, la lotta per il consenso interno alle comunità cristiane libanesi è passata in questi anni attraverso una miscela di argomentazioni anti-oligarchiche e di retoriche identitarie, in cui non sono mancati accenti di contrapposizione con migranti, rifugiati e settori vulnerabili della società libanese.

$\mathrm{Su}$ questo quadro complessivo sono planate le manifestazioni dell'autunno 2019, a cui hanno fatto seguito la crisi finanziaria del marzo 2020, la pandemia di Covid-19 e l'esplosione nel porto di Beirut del 4 agosto 2020. Lo sfaldamento del quadro politico e il collasso economico lasciano presagire una evidente insostenibilità dell'assetto esistente, ma senza che sembri possibile delineare al momento quali potrebbero essere gli scenari venturi.

${ }^{57}$ In questa sezione conclusiva riprendo una serie di considerazioni già svolte altrove. Si veda F. Mazzucotelli, Destra cristiana in Libano, l'offensiva è virulenta, in "il manifesto", 31 agosto 2019, https://ilmanifesto.it/destra-cristiana-in-libano-loffensiva-e-virulenta/ (ultimo accesso: 31 agosto 2021). 


\section{Appendice ${ }^{58}$}

1. Liste ammesse alla ripartizione dei seggi nel collegio Monte Libano 1 (Jbeil-Kisrawan)

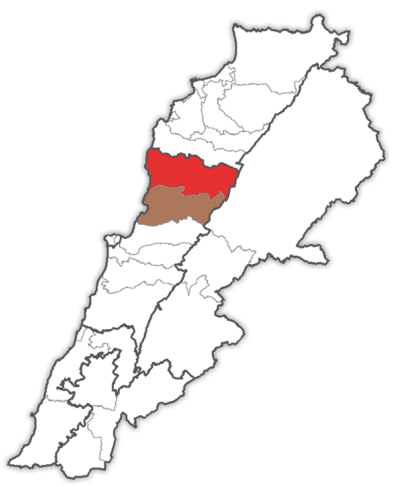

"Libano forte" (Movimento patriottico libero): 54'544 voti validi, 5 seggi.

"Cambiamento reale" (Forze libanesi): 26’980 voti validi, 2 seggi.

"La decisione spetta a noi" (Falangi libanesi, notabilato locale): 18'553 voti validi, 2 seggi.

2. Liste ammesse alla ripartizione dei seggi nel collegio Beirut 1 (in grigio nella mappa)

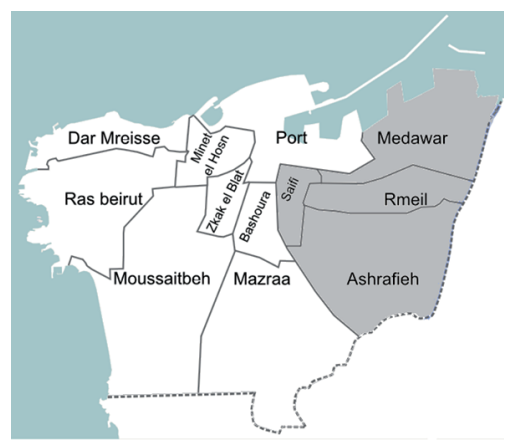

"Beirut I forte" (Movimento patriottico libero, partito armeno Tashnag, partito armeno Hunchak, con il sostegno esterno del Movimento per il futuro): 18’373 voti validi, 4 seggi.

"Beirut Prima" (Forze libanesi, Falangi libanesi, partito armeno Ramgavar, notabilato locale): 16'772 voti validi, 3 seggi.

"Siamo tutti connazionali" (società civile, progressisti): 6'842 voti validi, 1 seggio.

${ }^{58}$ I dati riferiti alle elezioni parlamentari del 2018 sono tratti dal sito del ministero degli interni libanese. (http://www.interior.gov.lb/AdsDetails.aspx?ida=281, ultimo accesso: 11 agosto 2021). Le mappe sono tratte da WikiCommons. I colori dei distretti all'interno di ciascun collegio elettorale non hanno alcun tipo di correlazione con le liste. 
3. Liste ammesse alla ripartizione dei seggi nel collegio Nord Libano 3 (Zgharta, Bsharri, Batrun, Kura)

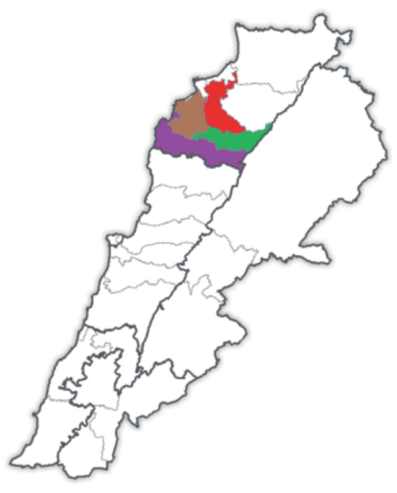

"Con noi per il nord e per il Libano" (Movimento mardaita, Partito nazionale sociale siriano, notabilato locale): $40^{\prime} 788$ voti validi, 4 seggi.

"Il battito forte della repubblica" (Forze libanesi, Falangi libanesi): 37'376 voti validi, 3 seggi.

"Nord forte" (Movimento patriottico libero, con il sostegno esterno del Movimento per il futuro): 33'342 voti validi, 3 seggi.

4. Liste ammesse alla ripartizione dei seggi nel collegio Monte Libano 2 (Matn)

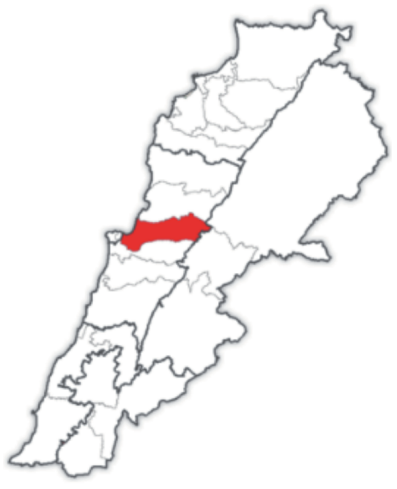

"Matn forte" (Movimento patriottico libero, partito armeno Tashnag, Partito nazionale sociale siriano): 38'897 voti validi, 4 seggi.

"Il battito del Matn" (Falangi libanesi): 19'003 voti validi, 2 seggi.

"Lealtà al Matn" (notabilato locale, dissidenti del Partito nazionale sociale siriano): $13^{\prime} 779$ voti validi, 1 seggio.

"Il Matn cuore del Libano" (Forze libanesi, partito armeno Ramgavar): 13'138 voti validi, 1 seggio. 


\section{Liste ammesse alla ripartizione dei seggi nel collegio Beqaa 1}

\section{(Zahlah)}

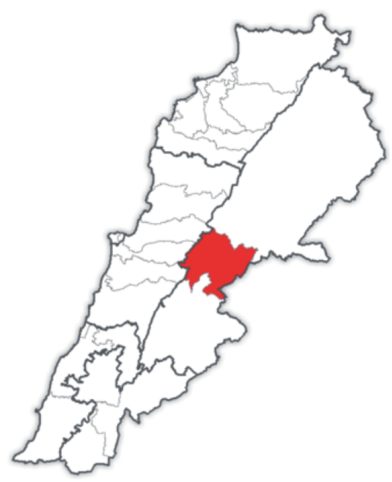

"Zahlah per tutti" (Movimento patriottico libero, Movimento per il futuro): 36'391 voti validi, 3 seggi.

"Zahlah, la scelta e le decisioni" (Hezbollah, notabilato locale, Partito nazionale sociale siriano): 23'546 voti validi, 2 seggi.

"Zahlah è la nostra causa" (Forze libanesi, Falangi libanesi): 18’702 voti validi, 2 seggi.

\begin{abstract}
A binary understanding based on the clash between the "8 March" and "14 March" coalitions does not adequately reflect the political processes within the Lebanese Christian arena between 2005 and 2019. Despite an increasing polarization after 2005 , in connection with the ongoing conflicts in the region, another dimension can be observed. Mass-based parties are confronted with the client-based politics of local notables, who often draw their legitimacy from family ties and personal fortunes. Mass parties deploy populist and charismatic arguments in order to bolster their status against the notables and their allegedly elitist politics. The cult of the leader is often based on a religious symbolism that draws heavily from the local Christian tradition, in particular from the concepts of
\end{abstract}

martyrdom and sainthood. What all major actors share is an idea of "Christian nationalism", which grounds the foundations of Lebanon's national identity in its Christian roots, and claims a sort of historical and political preeminence for Lebanese Christians.

This article shows how nationalist and sectarian discourses became stronger between 2005 and 2019. On the one hand, these arguments were deployed in order to strengthen a territorial bordering of the Christian-majority areas against the "external Other" (Muslims, Palestinians, nomads, migrants and Syrian refugees). On the other hand, these arguments were used to marginalize the "internal Other" (all those who questioned a homogenized Lebanese Christian society). 